\title{
Classification Performance of TM Satellite Images
}

\author{
Laith A. Al Ani 1,*, Hasan S. Al Tahir ${ }^{1}$ \\ ${ }^{1}$ Departments of Physics, Al-Nahrain University, Jadiriya 64055, Baghdad, Iraq
}

\begin{abstract}
Articles Information
Received:

29, 03, 2016

Accepted:

05, 04, 2016

Published:

01, March, 2020

\section{Keywords:}

Supervised classification-method

Maximum likelihood

Principle component analysis

Abstract

In the present work, the remotely sensed images had gone through different stages before it become ready to be classified, firstly by transforming the raw images by using principle component analysis method (PCA). Applying PCA has shown that the first three bands gives $97.86 \%$ of the overall information that has been provided from the scene. RGB coloring method is adopted as an unsupervised method to classify the scene according to the RGB color combination for three principle component images, this unsupervised method guide us to a better selection of the ROI (region of interest), it's becoming more clarity. Histogram equalization method has been used to enhance the colored bands. The results showed that the selecting of ROI from the original TM gave classification accuracy (85.84\%), whereas, after applying the RGB coloring model, the accuracy raised to (90.13\%). The accuracy has been shown to be equal (97.08\%) after applying the enhancement method, and by applying the same methods on the PC- images the accuracy of classification raised to become $(98.76 \%)$.
\end{abstract}

DOI: 10.22401/ANJS.23.1.09

* Corresponding author: laithalani2003@gmail.com

\section{Introduction}

The first problem in supervised satellite images classification is to extract a set of meaningful features from the image. Supervised classification of satellite image bands aimed to describe all of the classes spectrally $[1,2]$. The problem also comes from selecting the type and the number of bands for classification process. The widely used approach for selecting the best bands based on those bands that have the largest sum of squared principal axes that account for the largest total variance derived from the variance covariance matrix for the multi-spectral images of the scene [3]. The accuracy of a supervised image classification is a function of the region of interest (ROI) used in its generation. An appropriate choice of feature set is a vital factor in such application. As it will determine the ease and effectiveness with which subsequent segmentation and processing tasks may be performed [3, 4]. The primary purpose of this research is to classify multispectral Thematic Mapper (TM) satellite images using supervised classification. Unsupervised and supervised classification is adopted to achieve the classification purpose. The maximum likelihood (supervised classification method) classifier is generally considered to be the most powerful but is also considered the most computer intensive. According to this, Maximum likelihood (ML) algorithm is adopted to achieve this purpose

One of the most important concepts of pattern recognition in remote sensing is the definition of the classes into which the data to be categorized. The accuracy of a supervised classification analysis will depend upon the selection accuracy of the training area [5]. In this research K-L Transform will be applied to the original bands to create the principal component images. Color model like RGB model will be applied to the original bands and the first three components to create a colored image (Unsupervised classification method). This colored image will be employed for determining the training area

Some of enhancement techniques like histogram equalization will be applied to the created color image to improve the selection of the training area visually and will be reflected in the classification accuracy.

\section{The study Area}

The study remotely sensed imagery was presented by the Iraqi Geological Survey organization. The imagery was captured by the Landsat 8 (Worldwide Reference System (WRS-2) 


\section{Al-Nahrain Journal of Science}

ANJS, Vol.23 (1), March, 2020, pp. $62-68$

path 169 row 37), the provided scene is for Baghdad state on the 25th of September 2015. The scene consists of six bands as shown in figure 1 in $1003 \times 989$ pixels with an 8 bit gray. The data corrected and projected to UTM zone $38 \mathrm{Z}$. This area was well suited for the study as it contains various geographical land use and land cover, it contains Deep Water, Population, Trees, River, Streets, and various lands (Harvested lands, Barren Lands, Herbals Lands).

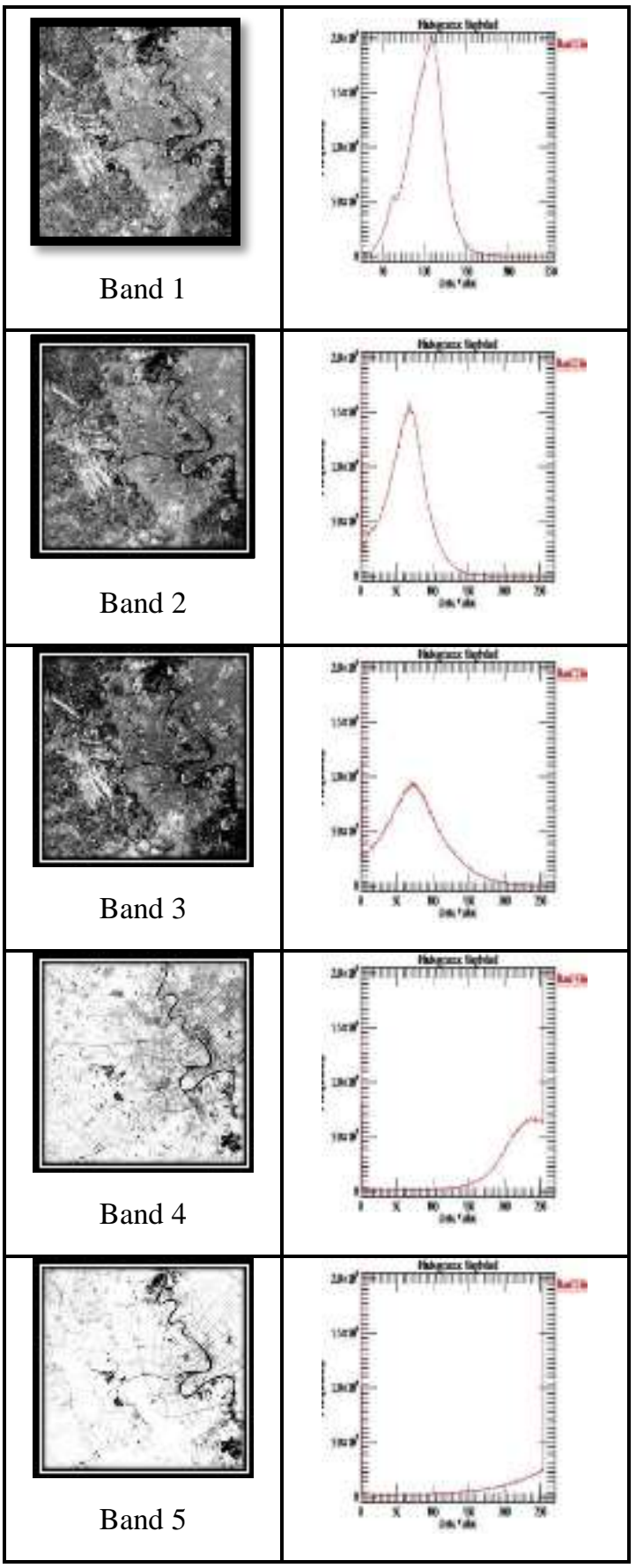

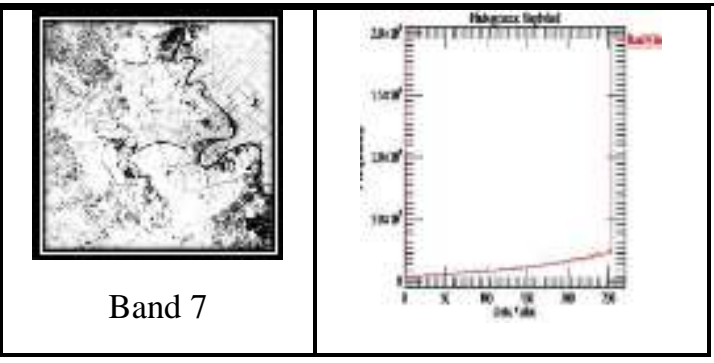

Figure 1. TM bands and its histogram distribution plot for Baghdad state with Landsat 8

To achieve the process of classification using ML method, it is important to choose adequately the training area for the classes in scene bands. It is clear from these bands that the quality of these bands seems to be poor and this affect the selecting the training area correctly. Now the coloring method must be applied to help the user to optimize the selection of the region of interest (ROI). The RGB coloring method is adopted for this purpose as an unsupervised classification to make the differentiation between different classes more clarity. The RGB color transforms require three bands for input. The RGB color transform uses this input to convert three-band red, green, blue (RGB) images to one of several specific color spaces. There will be many choices for selecting the three bands between the six bands, but the traditional method of choosing the three bands comes from choosing the three bands which have the largest variance value as shown in figure 2 .

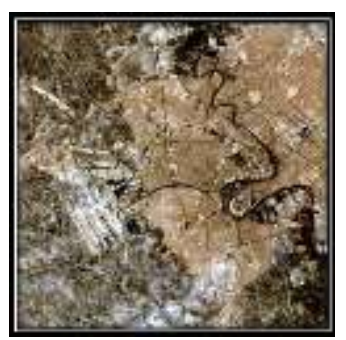

(a)

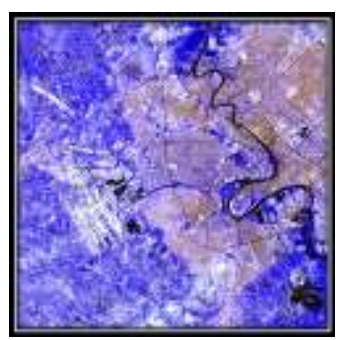

(b)
Figure 2. two color images from Baghdad using the RGB model from the bands which have the first two largest variance value $a$ and $b$ (bands $7,5,3$, sum of variance $=10337.8$ ) and (bands $7,5,4$, sum of variance $=10090.6$ ) respectively.

\section{Supervised classification}

Image classification is the process of assigning pixels or the basic units of an image to classes. It is likely to assemble groups of identical pixels found in remotely sensed data, into classes that match the 


\section{Al-Nahrain Journal of Science}

ANJS, Vol.23 (1), March, 2020, pp. $62-68$

informational categories of user interest by comparing pixels to one another and to those of known identity [6, 7]. The first step in supervised classification is to identify examples of the information classes of interest in the image. This stage is often called signature analysis and may involve developing a characterization as simple as the mean or the range of reflectance's on each band, or as complex as detailed analyses of the mean, variances and co-variances over all bands. Once a statistical characterization has been achieved for each information class, the image is then classified by examining the reflectance's for each pixel and making a decision about which of the signatures it resembles most [8, 9]. The quality of a supervised classification [6] depends on the quality of the training sites. All the supervised classifications usually have a sequence of operations that must be followed. 1. Defining of the Training Sites. 2. Extraction of Signatures. 3. Classification of the Image. The training sites are done with digitized features. Usually two or three training sites are selected. The more training site is selected, the better results can be gained. This procedure assures both the accuracy of classification and the true interpretation of the results. After the training site areas are digitized then the statistical characterizations of the information are created. These are called signatures. Finally the classification methods are applied $[6,7]$.

\section{Theoretical Basics}

The image processing is a vital stage for identifying and clarify the remote sensed image. This stage composed of three steps [5].

a. Image transformation:

The multispectral or vector character of most remote sensing image data renders it amenable to spectral transformations that generate new sets of image components or bands. These components then represent an alternative description of the data, in which the new components of a pixel vector are related to its old brightness values in the original set of spectral bands via a linear operation [5]. The transformed image may make evident features not discernable in the original data or alternatively it might be possible to preserve the essential information content of the image with a reduced number of the transformed dimensions. The last point has significance for displaying data in the three dimensions available on a color monitor or in color hardcopy, and for transmission and storage of data [10]. One of the most important transformation method is the Principle Component Analysis (PCA), which is based on the fact that neighboring bands of hyper spectral images are highly correlated and often convey almost the same information about the object [11]. The analysis is used to transform the original data so to remove the correlation among the bands. In the process, the optimum linear combination of the original bands accounting for the variation of pixel values in an image is identified [12, $13]$.

\section{b. Image enhancement:}

Histogram equalization is a common technique for enhancing the appearance of images. For continuous functions, the intensity (gray level) in an image may be viewed as a random variable with its probability density function (PDF) [14]. The PDF at a gray level $\mathrm{r}$ represents the expected proportion (likelihood) of occurrence of gray level $r$ in the image. A transformation function has the form:

$$
s=T(r)=(L-1) \int_{0}^{r} p_{r}(w) d w
$$

where $\mathrm{w}$ is a variable of integration.

\section{c. Image classification:}

Maximum Likelihood algorithm is one of the most supervised classification method that has been used with remote sensing image data to assign an unknown pixel to one of these classes [7]. Maximum likelihood rule a statistical decision criterion to assisting the classification of overlapping signatures; pixels are assigned to the class in which they have the highest probability of being a member. To overcome this problem, the widely used approach to this problem is through the use of principal component images [15]. By applying the principle component analysis the multispectral data set is compressed into one or two PC images, since the first few images contain essentially all information present in the original spectral bands. Moreover, the principle component images are spectrally uncorrelated. Table 1 and figure 3 shows the eigenvalue of the PC's, and the resulting components.

Table 1. Eigenvalue and relative eigenvalue of the covariance matrix and the accumulative variance.

\begin{tabular}{llll}
\hline P C & EV & R. E. V. & A. V. \% \\
\hline PC1 & 10279.90 & 0.7495 & $74.95 \%$ \\
PC2 & 1908.46 & 0.1391 & $88.86 \%$ \\
PC3 & 1234.66 & 0.0900 & $97.86 \%$ \\
PC4 & 223.37 & 0.0163 & $99.49 \%$ \\
PC5 & 61.72 & 0.0045 & $99.94 \%$ \\
PC6 & 8.42 & 0.0006 & $100 \%$ \\
\hline
\end{tabular}




\section{Al-Nahrain Journal of Science \\ ANJS, Vol.23 (1), March, 2020, pp. $62-68$}

The objective is to match the spectral classes in the data to the information classes of interest. It is the analyst's job to decide on the utility of the different spectral classes and their correspondence to useful information classes. The RGB method will be applied to the first three components and after that the histogram equalization method well be apply to enhance the colored image for a better selection of the region of interest.

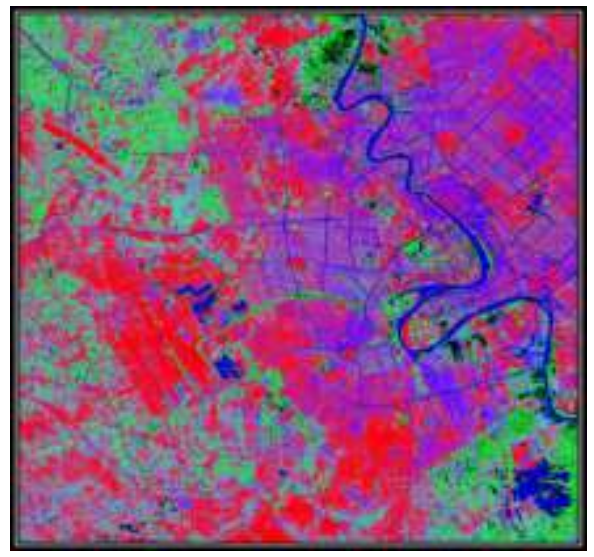

Figure 3. Color image constructed from enhanced principal component images using RGB model.

In the taken scene seven classes were adopted which represent distinguishable classes and used to classify this scene using the ML supervised method. These classes chosen from figure 3 and projected on one of the original bands as can be seen in figure 4 .

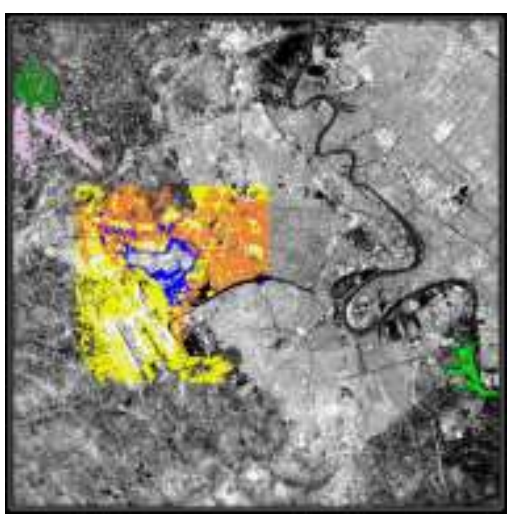

Class 1

Class 2

Class 3

Class 4

Class 5

Class 6

Class 7

Figure 4. The seven selected regions (ROI) as training classes for Baghdad scene

The presented results started by applying ML method to the original bands and to the enhanced color image and the principal component images. It should be mentioned that the process of classification applied to the original bands except the case where the classification method is directly applied to principal component images). The selected classes labeled relative to the real word classes on the earth are shown in table 2 .

Table 2. The selected classes labeled relative to the real word classes on the earth

\begin{tabular}{lcc}
\hline Class1 & Deep Water & $\mathbf{\square}$ \\
Class2 & Harvested Lands \\
Class3 & Population & Trees \\
Class4 & River And Streets \\
Class5 & Barren Lands \\
Class6 & Herbals Lands \\
Class7 & \\
\hline
\end{tabular}

Figure 5 presents the result of supervised classification for the original bands of the scene. The regions of interest are chosen visually for Baghdad scene.

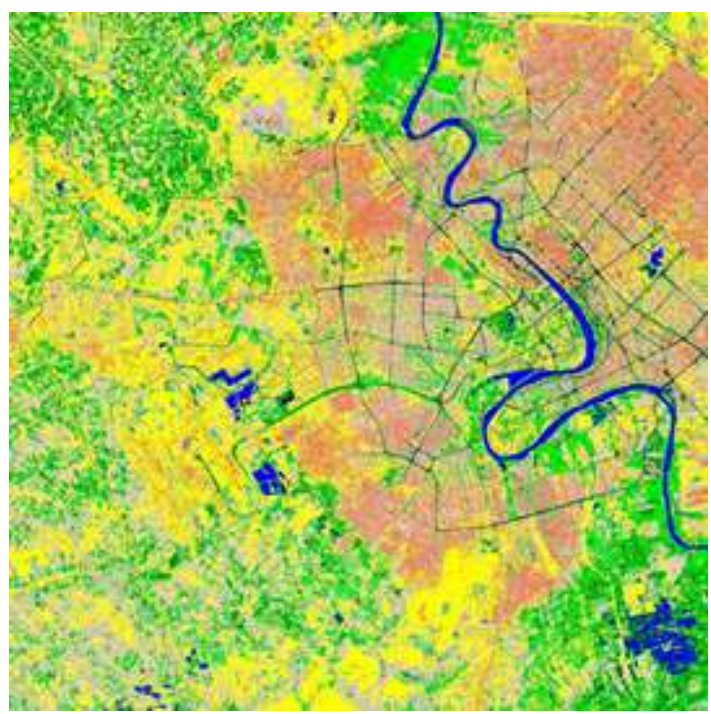

Figure 5. The result of the supervised classification for Baghdad scene.. The overall accuracy is about (85.84\%).

Figure 6 presents the result of the supervised classification using the six original bands after applying the RGB model to the bands which have the largest variance value. 


\section{Al-Nahrain Journal of Science}

ANJS, Vol.23 (1), March, 2020, pp. $62-68$

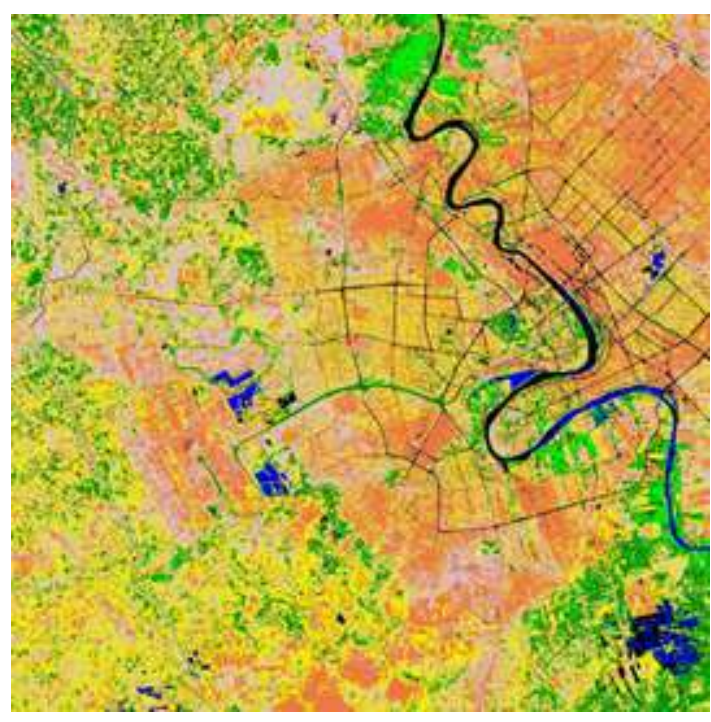

Figure 6. result of the supervised classification using the six original bands after applying the RGB model to the bands which have the largest variance value. The overall accuracy is about $(90.13 \%)$.

Figure 7 presents the result of supervised classification for the original bands. The regions of interest are chosen for the scene after applying histogram equalization on the RGB model to the bands which have the largest variance value.

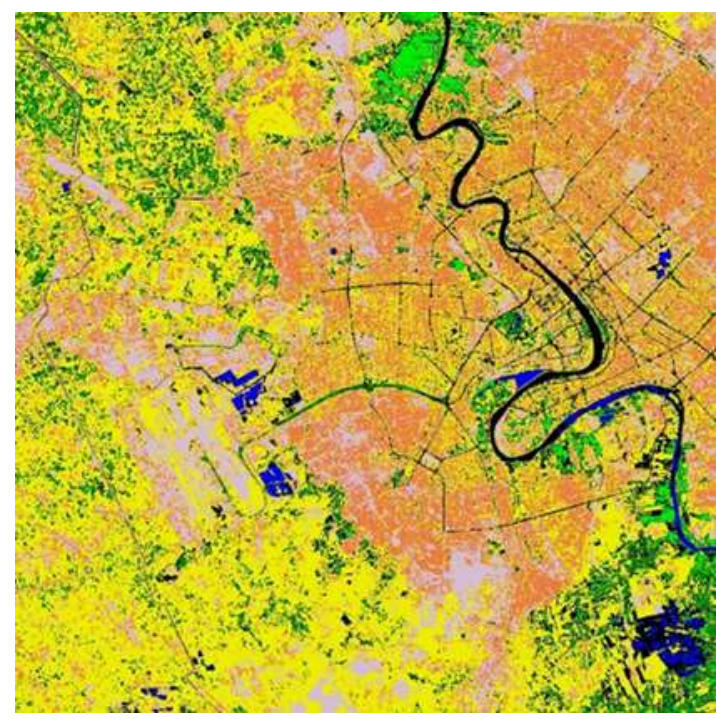

Figure 7. The result of the supervised classification. The regions of interest are chosen after applying histogram equalization on the RGB model to the bands which have the largest variance value. The overall accuracy is about (97.08\%).
Figure 8 presents the result of supervised classification for the principal component images.

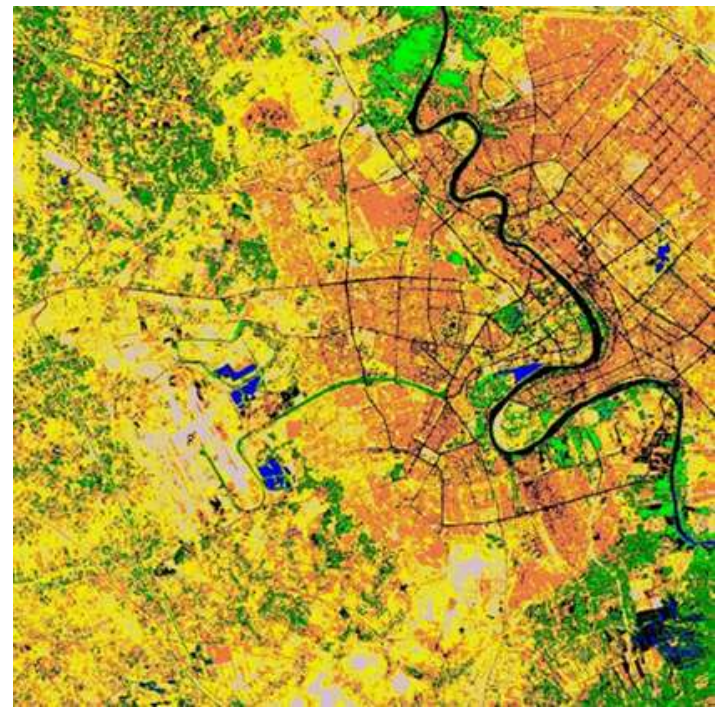

Figure 8. the result of the supervised classification using the six original bands (overall accuracy PC's (98.86\%)).

The regions of interest are selected from the color image of Baghdad scene constructed from the first three principal component images using the RGB model. the result showed that the overall accuracy is about $98.86 \%$ of the seven training samples selected from the principle components. It should be mentioned that all this information are time dependent, because of the changes in the nature of the land cover from a certain time to another. Table 3 summarize the results of the overall accuracy in supervised classification (maximum likelihood method) for all cases, which have been adopted in this study. 


\section{Al-Nahrain Journal of Science}

ANJS, Vol.23 (1), March, 2020, pp. $62-68$

Table 3. results of the overall accuracy in supervised classification (maximum likelihood method) for all cases, which have been adopted in this study.

\begin{tabular}{|c|c|c|c|c|}
\hline No & Type of ban & ents & Selected bands or components & Overall accuracy \\
\hline \multirow{4}{*}{1} & \multirow{4}{*}{\multicolumn{2}{|c|}{ Principal component }} & $(1,2,3)$ & $98.86 \%$ \\
\hline & & & $(1,2,3,4)$ & $98.96 \%$ \\
\hline & & & $(1,2,3,4,5)$ & $98.86 \%$ \\
\hline & & & $(1,2,3,4,5,6)$ & $98.74 \%$ \\
\hline \multirow{8}{*}{2} & \multirow{8}{*}{ Original bands } & \multirow{4}{*}{ Visually } & $(1,2,4)$ & $90.34 \%$ \\
\hline & & & $(1,2,4,5)$ & $89.14 \%$ \\
\hline & & & $(1,2,3,4,5)$ & $90.03 \%$ \\
\hline & & & $(1,2,3,4,5,7)$ & $90.13 \%$ \\
\hline & & \multirow{4}{*}{ Statistics } & $(7,5,3)$ & $74.28 \%$ \\
\hline & & & $(7,5,3,4)$ & $68.54 \%$ \\
\hline & & & $(7,5,3,4,2)$ & $82.89 \%$ \\
\hline & & & $(7,5,3,4,2)$ & $90.13 \%$ \\
\hline \multirow{4}{*}{3} & \multirow{4}{*}{\multicolumn{2}{|c|}{ Enhanced original bands with histogram equalization }} & $(7,5,3)$ & $82.41 \%$ \\
\hline & & & $(7,5,3,4)$ & $79.65 \%$ \\
\hline & & & $(7,5,3,4,2)$ & $89.33 \%$ \\
\hline & & & $(7,5,3,4,2)$ & $97.08 \%$ \\
\hline
\end{tabular}

\section{Conclusions}

From the results its can be conclude the following remarks. Improving the selection of the training area visually plays an essential role in increasing the accuracy of supervised classification. Dealing with principal component images solved the problem for choosing of which bands best for classification purpose. This is so because the principal component images are arranged from the component of high energy (contain most of the details, the first principal component image) to the lowest one. The result of classification accuracy is very high with principal component image, but still the classification process with original bands is better when we are dealing with principal component images. The result of classification accuracy with the original bands is enhanced from $68.5 \%$ to $97.08 \%$ and became very comparable with the result of classification accuracy with the principal component images $98.86 \%$.

\section{References}

[1] Foody, G. M.; Mathur A.; "Toward intelligent training of supervised image classifications: directing training data acquisition for SVM classification"; Remote Sensing of Environment 93, 107-117, 2004.

[2] Foody, G. M.; Mathur A.; "The use of small training sets containing mixed pixels for accurate hard image classification: Training on mixed spectral responses for classification by a SVM"; Remote Sensing of Environment 103, 179-189, 2006.

[3] Al-Sebahi, A.; Al-Ani, L.; "Classification of Digital Satellite Images Using Selective Bands and Principal Component Images" M. Sc. Thesis, AlNahrain University, College of Science, 2002.

[4] Zhou, H.; Zhang, Y.; Yu, Z.; "Image classification based on region of interest detection", Proc. SPIE 9813, MIPPR: Pattern Recognition and Computer Vision, Vol. 9813, 2015.

[5] Al-Ani, L.; "Classification of Digital Satellite Images", Ph.D. Thesis, Al-Nahrain University, College of Science, 1996.

[6] Palaniswami, C.; Upadhyay, A. K.; Maheswarappa, H. P.; "Spectral mixture analysis for sub pixel classification of coconut", Current Science, 91(12), 1706 -1711, 2006.

[7] Perumal, K.; Bhaskaran, R.; "Supervised Classification Performance of Multi-Spectral Images”. J. Computing 2, 124-129, 2010.

[8] David, C.; "Environmental Science and Information Application Technology", CRC press, 2015.

[9] Langer, H.; Falsaperla, S.; Masotti, M.; Campanini, R.; Spampinato, S.; Messina, A.; "Synopsis of supervised and unsupervised pattern classification techniques applied to volcanic tremor data at $\mathrm{Mt}$ Etna, Italy", Geophys. J. Int. 178, 1132-1144, 2009.

[10] Richards, J. A.; "Multispectral Transformations of Image Data", Springer-Verlag Berlin Heidelberg, 2013. 


\section{Al-Nahrain Journal of Science}

ANJS, Vol.23 (1), March, 2020, pp. $62-68$

[11] Al-Ani, L.; Al-Ani, A.; Alyaa, H. A.; "Principal Component Analysis of Multi-Temporal Image Pairs", Iraqi Journal of Science 47(1), 220-226, 2006.

[12] Rodarmel, C.; Sha, J.; "Surveying and Land Information Systems", Vol. 62, No. 2, 115-000, 2002.

[13] Keerthana, P.; Sivasankar, A.; "The Impact of Lossy Compression on Hyper spectral Data Adaptive Spectral Un-mixing and PCA Classification”, IJISM, E 1, 35-37, 2013.
[14] Maini, R.; Aggarwal, H.; "A Comprehensive Review of Image Enhancement Techniques”. Journal of computing 2, 8-13, 2011.

[15] Korgaonkar, G. S.; Sedamka, R. R.; Bhandari, K.; "Hyper spectral Image Classification on Decision level fusion", International Conference \& Workshop on Recent Trends in Technology, (TCET), 2012. 